\title{
PROKLA-Redaktion
}

\section{Editorial: Die politische Krise in Europa und die Reorganisation der bürgerlichen Kräfte}

„Noch vor einem Jahrzehnt glaubten viele, sie sei am Schluss ihrer Weisheit und Wirksamkeit angelangt ...: die Theorie des Neo-Liberalismus" - so Elmar Altvater 1981 in der PROKLA 44. Die ökonomischen und politischen Folgen der Krise 1929ff. brachten das Laisser-faire in die Defensive. Mit den Klassenkämpfen und sozialen Auseinandersetzungen in den 1960er und 1970er Jahren galt der Keynesianismus in den industriellen Zentren als die anerkannte wirtschaftspolitische Doktrin sowie die adäquate Form, den Widerspruch von Kapital und Lohnarbeit zu bearbeiten. „Lieber fünf Prozent Inflation als fünf Prozent Arbeitslosigkeit" - noch 1972 schien der Ausspruch des späteren Bundeskanzlers Helmut Schmidt Common Sense, wurde Geldwertstabilität für weit weniger wichtig gehalten als das Beschäftigungsniveau. Es kam bekanntlich anders - auch dank Helmut Schmidt, der die ersten Austeritätsmaßnahmen einleitete, Jahre vor Helmut Kohl. Mit Ronald Reagan in den USA, Margaret Thatcher in Großbritannien und Helmut Kohl in Deutschland wurde der sozialstaatlich verfasste Nachkriegskapitalismus jedoch endgültig angezählt. Rot-Grün unter Gerhard Schröder markierte mit der Agenda 2010 den vorläufigen Höhepunkt neoliberaler Politik in Deutschland. Die Linke hatte es über Jahre schwer, mit der Offensive von Kapitalinteressen und neuer Bürgerlichkeit einen politischen Umgang zu finden.
Angesichts der tief greifenden Krise des Kapitalismus, die ihren Ausgangspunkt zwar 2006 auf dem US-Immobilienmarkt hatte, sich aber 2007 über die globalen Finanzmärkte weltweit ausbreitete, zeigten sich viele Linke guten Mutes: Die Hegemonie des Neoliberalismus habe Risse bekommen, in die das "Neue" eindringen könnte, das überall und dank des globalen Widerstands gegen die sozialen und politischen Zumutungen bereits aufblitze (vgl. PROKLA 177).

In Europa repräsentierte für viele Syriza die Hoffnung politischer Alternativen, nachdem die Krise schließlich in die sogenannte Eurokrise (2009ff.) mündete, auf die die Euro-Länder unter deutscher Führung mit Austerität antworteten, während Angela Merkel die „marktkonforme Demokratie“ ausrief. Die Linkspartei in Griechenland, aber auch Podemos in Spanien machten vielen Hoffnung, dass die neoliberale Alternativlosigkeit in eine Sackgasse geraten war. Seit Juli 2015 wissen wir, dass es anders kam. Selbst wenn die Geschichte noch nicht zu Ende ist.

In einigen der letzten PROKLA-Ausgaben sind wir vor allem auf die ökonomische Dimension der Krise (PROKLA 157), die Krise der Wirtschaftswissenschaften (PROKLA 164), die EU und den Euro (PROKLA 168) und den „Krisengewinner Deutschland" (PROKLA 166) eingegangen. Dass Deutschland Krisengewinner ist, ist eine Voraussetzung dafür, dass eine 
breite Zustimmung für die Austeritäts- und Dominanzpolitik der Bundesregierung organisiert werden kann. Auch viele Texte außerhalb der jeweiligen Schwerpunkte hatten Griechenland zum Thema. In der vorliegenden PROKLA geht es vor allem darum, wie sich die Krise politisch in der Reorganisation der bürgerlichen Kräfte ausdrückt und fortschreibt.

Die Krise hatte nicht nur ökonomische, sondern auch politische und ideologische Folgen. Sie zerrüttete die Profitaussichten und veränderte die Reproduktionsbedingungen des Kapitals insgesamt. Diese Veränderungen trafen nicht alle Kapitale gleichermaßen. Entsprechend vielfältig sind die Reaktionen - etwa der Unternehmensverbände. Während Finanzvermögen (u.a. in Griechenland) gerettet wurden und die Troika-Politik in der Eurozone auf eine Tragfähigkeit der Staatsschulden und eine Schwächung der ArbeiterInnenklasse abzielt, unterminiert dieser Austeritätskurs gleichzeitig die Binnenwirtschaft der betroffenen Länder und stellt für das auf diese Binnenmärkte ausgerichtete Kapital ein Problem dar. Das exportorientierte Deutschland, für das der EU-Binnenmarkt nach wie vor der wichtigste Absatzmarkt ist, drängte trotzdem auf einen europaweiten Sparkurs - und konnte dank der wachsenden Bedeutung der nicht-europäischen Absatzmärkte (China, USA) diesen Kurs durchhalten. Die Instabilitäten etwa in China zeigen aber auch, wie wichtig Alternativen für das deutsche Kapital sind. Der vor allem vom deutschen Außenministerium vorangetriebene Iran-Deal erscheint damit ebenfalls in einem anderen Licht.

Der Titel von Altvaters 1981 verfasstem Aufsatz („Der gar nicht diskrete Charme der neoliberalen Konterrevolution“) spielte auf einen Film von Luis Buñuel von 1972 an: Der diskrete Charme der Bourgeoisie, in dem sechs RepräsentantInnen ihrer Klasse in einem fiktiven lateinamerikanischen Land zu einem Essen zusammenkommen wollen und sich aus verschiedensten Gründen ständig verpassen, aber - allen Abstrusitäten zum Trotz - an ihren Ritualen festhalten. Wie in Buñuels Filmen scheint die jetzige bürgerliche Welt gleichfalls aus den Fugen geraten zu sein. Trotz schwerer politischer Krise und vieler Widersprüche wirkt es jedoch, als hätten sich die bürgerlichen Kräfte in Europa vorerst reorganisiert und ihre Rituale der Austeritätspolitik sich verfestigt - nicht nur, aber auch dank der ordnungspolitischen Faust aus Berlin.

Für internationale Beobachter mag die Politik der Bundesregierung ebenso surreal erscheinen wie so manch ein Buñuel-Film. Weltweit renommierte Ökonomen wie Paul Krugman oder Thomas Piketty vertraten wiederholt öffentlich, auch in einem Brief an Bundeskanzlerin Angela Merkel, dass die Griechenland aufgezwungene Austeritätspolitik zu weiterer Verarmung und einer Destabilisierung der Eurozone führt. Selbst der Internationale Währungsfonds (IWF) positioniert sich in der Frage eines Schuldenschnitts inzwischen offen gegen die deutsche Bundesregierung. Deutschland hielt dennoch Kurs, mit zumindest passiver Unterstützung aus Frankreich. Der Ökonom und Berater des ehemaligen EU-Kommissionspräsidenten José Manuel Barroso, Philippe Legrain, schrieb in Foreign Policy: „Ja, kleine Länder wie die Slowakei und Finnland haben Deutschland zugestimmt. Aber ihre Stimmen sind letztlich nicht entscheidend. Aus Berliner Perspektive sind sie die nützlichen Idioten, um die engstirnigen deutschen Interessen zu decken. Um es klar zu sagen. Was Berlin und Frankfurt Griechenland angetan haben - genau das werden sie auch mit allen anderen machen." Und Shahin Vallée vom European Institute der London School of Economics schrieb in der New York Times: 
„Deutschland hat Frankreich signalisiert, dass es bereit war, alleine voran zu gehen." Deutschland hat in den letzten Jahren nicht unbedingt seine wirtschafts- und europapolitischen Positionen geändert, aber sein Gewicht in den Verhandlungen massiv vergrößert. Allen voran Frankreich ist nicht mehr in der Lage, Berlin auch nur ansatzweise substanzielle Kompromisse abzuringen. „Wir erleben gegenwärtig die innere Kolonialisierung Europas: Die politische Ökonomie des europäischen Imperialismus kehrt aufden Kontinent und, mehr noch, innerhalb der Grenzen der Europäischen Union zurück", so das Mitglied des PROKLA-Beirats, Stephan Lessenich, in der Süddeutschen Zeitung (27.7.2015). Die Ereignisse im Juli 2015 haben gezeigt, dass sich die Eurokrise zu einer tief greifenden politischen Krise transformiert hat - und Deutschland ist wieder wer.

Für das gegenwärtige Verständnis der Veränderung bürgerlicher Herrschaft in Europa wurden in den letzten Jahren verschiedene Begriffe eingebracht: „kollektiver Bonapartismus" (Hauke Brunkhorst) oder "autoritärer Wettbewerbsetatismus“ (Lukas Oberndorfer). Auch der schon vor Jahren von Colin Crouch entwickelte Begriff der „Postdemokratie“ wurde herangezogen. Im Hinblick auf Mittel- und Osteuropa, aber auch Griechenland, wird oft von „Oligarchie“ gesprochen. Anhand der postsozialistischen Transformationsprozesse in Russland, der Ukraine, Serbien, der Tschechischen Republik und der Slowakei geht Joachim Becker in seinem Beitrag für diese PROKLA der Frage nach, was die Oligarchie als eine Form bürgerlicher Herrschaft auszeichnet, deren Elemente zunehmend auch in Westeuropa anzutreffen sind.

Bei der Frage nach der politischen Form der Absicherung bürgerlicher Herrschaft darf nicht vergessen werden, dass es sich im
Kern um einen sozialen Konflikt handelt, d.h. um die Frage, wie dieser bearbeitet und soziale Ungleichheit reproduziert wird. Jens Beckert und Wolfgang Streeck stellten 2011 eine zentrale Ausgangsfrage: „Nachdem die Zuwächse des Sozialprodukts während der vergangenen dreißig Jahre vornehmlich den oberen Bevölkerungsschichten zugutekamen, stellt sich in der Schuldenkrise die Frage, ob und mit welchen Mitteln die Wohlhabenden versuchen werden, ihre Position auch um den Preis einer massiven sozialen und politischen Krise zu verteidigen" (FAZ, 20.8.2011). In den vergangenen Jahren ist deutlich geworden: Das Kapital, die Vermögenden und die Gläubiger-Länder verteidigen ihre Pfründe und ihren Handlungsspielraum - und dabei scheint ihnen inzwischen fast jedes Mittel recht, um die Frage von Beckert/Streeck in Kurzform zu beantworten. Eine Lösung der Krise zulasten der Vermögenden wird nach wie vor vehement blockiert. Bis auf wenige Ausnahmen ist es der Linken nicht gelungen, eine Bearbeitung der Krise im Sinne wirksamer Umverteilung von oben nach unten entlang der Klassenspaltung auf die Tagesordnung zu setzen. Demgegenüber konnten die bürgerlichen Kräfte erfolgreich die Lohnabhängigen verschiedener Länder gegeneinander ausspielen und ihre Strategie durch nationalistische Diskurse stützen.

Dabei gelingt es rechtspopulistischen Parteien wie den Wahren Finnen oder dem Front National, insbesondere das konservative Lager vor sich herzutreiben, das zu immer weniger Zugeständnissen auf europäischer Ebene bereit ist. In Deutschland hingegen ist die rechte Alternative für Deutschland (AfD) aufgrund eigener Widersprüche zwar blockiert und als politische Kraft in Auflösung begriffen. Der Schock des rasanten Aufstiegs einer rechten Partei mit bürgerlichem Anstrich scheint der CDU aber so tief in den Knochen zu 
sitzen, dass sie sich, unterstützt von der SPD, die Politik der ,eurokritischen“ Partei selbst aneignet. Schäubles Vorgehen und der Vorstoß, Griechenland aus dem Euro zu werfen, ist die Fortführung der AfDPolitik mit anderem Parteibuch - auch wegen des erheblich innerparteilichen Widerstands gegen weitere Kredite für Griechenland, der mit den rechten „eurokritischen" Positionen der AfD sympathisiert und selbst Angela Merkel ungewohnt heftig schwächt. Die autoritäre Politik wird vor allem in Deutschland von einer breiten Mehrheit getragen. Schäuble ist so beliebt wie nie. Viele sozialwissenschaftliche Analysen gehen davon aus, dass der Zuspruch für autoritäre Politik, Entsolidarisierung und „Menschenfeindlichkeit" ihren Grund in der Prekarisierung und Verunsicherung breiter Schichten der Bevölkerung haben, für die zwar rechtspopulistische, aber kaum emanzipatorische oder linke Deutungsangebote gemacht werden können. Dem widerspricht Thomas Goes in diesem Heft und zeigt anhand qualitativer Interviews, dass ein "linker Populismus“ durchaus möglich ist.

Durch massiven staatlichen Einsatz wurde eine Entwertung des Kapitals verhindert. Welche politischen Widersprüche ergeben sich daraus und wie artikulieren sie sich in den bürgerlichen und konservativen Parteien im Verlauf der Krise? Darauf gehen John Kannankulam und Fabian Georgi in ihrem Beitrag zu diesem Heft ein und zeigen, dass die in der Bundesregierung dominante ,autoritärneoliberale" Fraktion im Sommer 2012 einen Kurswechsel einleitete, indem sie mit ordoliberal-konservativen Prinzipien brach und ihren erbitterten Widerstand gegen Schuldenvergemeinschaftung und expansive Geldpolitik abschwächte. Die daraus entstehenden Widersprüche führten zur Verselbstständigung eines Teils des ordoliberal-konservativen Lagers und ermöglichten die Gründung der AfD, was jedoch neue Konflikte im bürgerlichen Lager hervorrief und die weitgehend kompromisslose Austeritätslinie der Bundesregierung in den Auseinandersetzungen mit der griechischen Regierung neuerlich verhärtete.

Auch die ökonomische Krise setzt sich bis dato fort und liefert einen Resonanzboden für die unterschiedlichsten Phänomene wie rechten Populismus, bürgerliche Moralkampagnen, Antifeminismus, sozialen Populismus und verschärfte Repression, auch wenn sich diese nicht unmittelbar als notwendige Folgen aus der ökonomischen Krise ableiten oder auf sie reduzieren lassen. Während Felix Syrovatka die Erfolgsbedingungen und Strategien des Front National thematisiert, geht Daniel Keil der sozialen Basis und den ideologischen Elementen des Phänomens Pegida nach, gewissermaßen dem außerparlamentarischen Arm der AfD. Pegida und seinen diversen Ablegern gelangen die größten rechten Massenmobilisierungen seit Jahren, die die Vorzeichen der gegenwärtigen rassistischen Anschläge und Angriffe auf Flüchtlinge und Flüchtlingsunterkünfte darstellen. Spätestens nach der Niederlage Syrizas im Juli 2015 ist unbezweifelbar, dass nicht die Linke, sondern vor allem die Rechte in Europa gestärkt aus den vergangenen Krisenjahren hervorgeht.

Michael Heinrich kritisiert in seinem Einspruch die verbreitete These, der Euro sei eine Fehlkonstruktion und hält demgegenüber fest, dass der in den Maastricht-Vertrag eingebaute Zwang zur Austeritätspolitik durchaus so gewollt ist. Insofern geht die in Teilen der Linken verbreitete Auffassung, EU und Euro seien doch 'eigentlich' eine gute Sache, nur in den Händen der falschen Politiker, an der Realität vorbei. Gegen das Zusammenspiel von EZB und deutscher 
Regierung haben ökonomisch schwächere Euro-Mitglieder keine Chance.

Viele Fragen bleiben leider offen, Fragen, die an anderer Stelle oder vielleicht in folgenden Heften thematisiert werden müssen.

Außerhalb des Schwerpunkts setzt Christian Lotz seine Kritik an postmarxistischen Strömungen aus PROKLA 176 fort und geht auf die neuere Kritische Theorie ein (Honneth, Habermas), die das Forschungsprogramm der Kritik der politischen Ökonomie verlassen hat und statt in der Gesellschaftskritik auf dem Feld der Moral nach letztgültigen Prämissen sucht - und nicht fündig werden kann. Selbst wenn der Text von Christian Lotz außerhalb des Schwerpunkts ist, so schließt sich der Kreis, schließlich stellt sich mit der Reorganisation der bürgerlichen Kräfte immer auch die Frage, wie sie zu kritisieren sind.

In seinem Besprechungsessay eröffnet Alex Demirović die Diskussion über die vollständige und ergänzte Neuübersetzung von Das Kapital lesen von Louis Althusser und seinen Mitarbeitern. Dabei referiert er nicht nur die lange Rezeptionsgeschichte zwischen Missverständnissen und Verdrängung, sondern macht deutlich, warum die Beiträge - gerade heute - für eine kritisch-marxistische Theorie der kapitalistischen Gesellschaftsformation ein noch unausgeschöpftes Potenzial bergen.

Etienne Schneider, Thomas Sablowski
und Ingo Stützle
(Für die Redaktion)

\section{PROKLA 181 Geopolitische Konflikte nach der "neuen Weltordnung" (Dezember 2015)}

Nur ein knappes Vierteljahrhundert nach ihrer Ausrufung durch George H.W. Bush befindet sich die „neue Weltordnung“ in Auflösung. Der Konflikt in der Ukraine, die (Bürger-)Kriege im Nahen Osten und der Aufstieg des sogenannten Islamischen Staat, die Weltwirtschaftskrise und die durch sie beschleunigten Verschiebungen zugunsten der BRICS-Staaten, die Krise in der EU, die Konflikte um natürliche Ressourcen und die ökologischen Krisenphänomene wie der Klimawandel haben die Vorstellung einer friedlichen globalen Entwicklung unter kapitalistischen Vorzeichen und unter US-amerikanischer Führung gründlich desavouiert. Es verstärken sich zudem sowohl Tendenzen der ökonomischen und politischen Vereinheitlichung des kapitalistischen Weltsystems als auch Tendenzen seiner zunehmenden Fragmentierung. Welche Art der Weltordnung sich gegenwärtig herausbildet, was also nach der „neuen Weltordnung" kommt, ist auch in der Diskussion keineswegs unumstritten. Offen ist ferner, welche Konfliktachsen dabei in den Vordergrund treten und welche Bündnisse sich herausbilden würden. Das Heft wird die geopolitischen Konflikte nach der "neuen Weltordnung“ empirisch wie theoretisch diskutieren. 